\title{
CUIDAR Y CRIAR EN COMUNIDADES ORIGINARIAS: UNA APUESTA DE INVESTIGACIÒN INTERCULTURAL ENTRE COLOMBIA-BRASIL
}

\section{CARING AND GROWING IN NATIVE COMMUNITIES: A BET FOR INTERCULTURAL RESEARCH BETWEEN COLOMBIA AND BRAZIL}

\section{Lorena Cardona Alarcón ${ }^{1}$}

Fundación Universitaria del Área Andina,

Bogotá- Colombia

\section{Eliane Rodrigues de Castro ${ }^{2}$}

Rede Pública Municipal de Juiz de Fora/ UFJF, (Minas Gerais)- Brasil

\footnotetext{
$1 \quad$ Estudiante del Doctorado en Literatura de la Universidad Tecnológica de Pereira, Colombia. Magister en Literatura de la Pontificia Universidad Javeriana de Bogotá. Licenciada en Lenguas Modernas de la Universidad de Caldas, Manizales. Docente e investigadora de la Fundación Universitaria del Área Andina. Miembro del Grupo de Investigación Kompetenz de la misma institución. Email: Icardona46@areandina.edu.co. ORCID: 0000-0001-7593-7285 2 Especialista em Geografia e Gestão do Territorio (UFJF). Licenciada em Geografia (UFJF). Professora da Rede Pública Municipal de Juiz de Fora. Professora da Secretaria de Educação do Estado do Río de Janeiro. Investigadora do Grupo de Pesquisas e Estudos em Geografia da Infancia (GRUPEGI/UFJF-UFF). Email: elianercastro01@ yahoo.com.br
}

\section{RESUMEN}

Este artículo es producto del intercambio académico y el diálogo intercultural entre investigadores de Brasil y Colombia, en el marco del proyecto Geografías del cuidado y la crianza desde la experiencia educativa en comunidades originarias de América Latina, una apuesta que adelantan la Fundación Universitaria del Área Andina (Colombia) y la Universidad Federal Juiz de Fora (Brasil), a través de sus Facultades 
de Educación y los grupos de investigación Kompetenz y GRUPEGI, respectivamente, como respuesta a los retos que los contextos socioculturales colombianos, brasileños y latinoamericanos, le demandan a la academia desde los saberes originarios que necesitan ser visibilizados para comprender las realidades diversas de nuestros niños y niñas.

PALABRAS CLAVE: comunidades originarias, interculturalidad, infancias, cuidado, crianza, geografía de la infancia.

\section{ABSTRACT}

This article is the product of academic exchange and intercultural dialogue between researchers from Brazil and Colombia, within the framework of the Geographies of Care and Parenting project from the educational experience in communities originating in Latin America; a bet advanced by the University Foundation of the Andean Area (Colombia) and the Federal University Juiz de Fora (Brazil), through its Faculties of Education and the Kompetenz and GRUPEGI research groups, respectively, in response to the challenges that the contexts Colombian, Brazilian and Latin American socioculturals demand the academy from the original knowledge that needs to be made visible to understand the diverse realities of our children.

KEYWORD: original communities, interculturality, childhood, care, parenting, childhood geography.

\section{RESUMO}

Este texto é o produto do intercâmbio acadêmico e do diálogo intercultural entre pesquisadores do Brasil e da Colômbia, no âmbito do projeto Geografias do Cuidado e Paternidade, a partir da experiência educacional em comunidades originárias da América Latina; uma aposta promovida pela Fundação Universitária da Área Andina (Colômbia) e Universidade Federal Juiz de Fora (Brasil), através de suas Faculdades de Educação e grupos de pesquisa Kompetenz e GRUPEGI, respectivamente, em resposta aos desafios que os contextos sociocultural colombianas, brasileiras e latino-americanas exigem da academia o conhecimento original que precisa ser tornado visível para entender as diversas realidades de nossos crianças.

PALABRAS CHAVE: comunidades originais, interculturalidade, infâncias, cuidados, criação, Geografia da Infância.

\section{Cuidar y criar en comunidades originarias: una apuesta de investigación intercultural entre Colombia-Brasil}

Nuestra diversidad cultural es una herencia, es preciosa y la diferencia de culturas encontrará sus propias formas para concretar lo establecido. Debemos profundizar y ampliar el diálogo global... puesto que tenemos mucho que aprender en la búsqueda colaboradora de la verdad y la sabiduría. Carta a la Tierra. (De Sousa, 2000).

"As crianças nascem em paisagens préexistentes, vivenciam territórios, lugares e outras dimensões espaciais que são expressões do espaço geográfico" (Lopes, 2016, p.2).

\section{INTRODUCCIÓN}

Los modelos educativos latinoamericanos se han caracterizado por validar, en la mayoría de los casos, la episteme occidental. Las culturas ancestrales, las comunidades originarias y sus saberes han estado históricamente excluidos de la academia. En casos particulares donde la presencia de etnias y movimientos indígenas ha sido importante, las universidades han creado programas curriculares y propuestas de investigación para favorecer a esas comunidades en ejercicios formativos. Sin embargo, se puede afirmar que, en general, la interculturalidad ha sido un concepto marginal y que la universidad latinoamericana no es una universidad pensada y concebida como intercultural. 
De allí que sea necesaria la incorporación de la interculturalidad $^{3}$ como una noción nuclear en el campo universitario e investigativo, para el acercamiento y reconocimiento de las prácticas culturales y saberes ancestrales propios de los comunidades originarias de Colombia, Brasil y América Latina, ya que desde una mirada de larga duración, las culturas locales han sido rezagadas y han perdido su posibilidad de preservación, debido a que se enfrentan, en primer lugar, a la fuerza de la cultura dominante o cultura oficial; en segundo lugar, a los flujos migratorios hacia las ciudades que han hecho abandonar las prácticas tradicionales, es decir, se ha provocado una ruptura significativa con sus raíces, lo cual puede catalogarse como una ausencia de enraizamiento y subalternización cultural.

En este sentido, la tarea de las universidades y la academia -en general- con los territorios diversos $^{4}$ y las comunidades originarias de esta parte del Planeta, es dar respuesta a sus realidades, referenciando las particularidades culturales del contexto y visibilizando sus tradiciones, sus prácticas, en suma, su identidad, en los ámbitos investigativo y de proyección social, más allá de los marcos conceptuales normalizados del mundo occidental, la teoría estática o el currículo, ya que comprender las epistemes de las comunidades originarias desde los marcos conceptuales tradicionales, sería una tarea contradictoria en sí misma, pues no cabría en un solo punto de vista, la complejidad que entraña entender la reivindicación de la tierra, la ancestralidad, los símbolos, las prácticas tradicionales, entre otros, y se confundiría

$3 \quad$ Entendida como "una apuesta política y cultural, que insta al reconocimiento de la diversidad en el continente latinoamericano, es un eje de interés en el marco de la construcción de propuestas investigativas que alimenten una perspectiva de academia más allá del horizonte epistémico de Occidente". (Cardona, L., Ramírez, M., Vergara, M. y Gutiérrez, A., 2017, p.25)

$4 \quad$ La idea del territorio puede considerarse como una especie del concepto genérico de derecho a la tierra, sólo que enriquecido con los elementos políticos e identitarios con que los pueblos étnicos interpretan su entorno." (Herreño, 2004) con una postura instrumental o una mirada reduccionista elaboradas desde Occidente.

De acuerdo con lo anterior, la academia y, por supuesto la investigación, deben superar la mirada monolítica del saber, la cual encumbra como legítimo el saber científico y expulsa las fuentes de la experiencia cultural, donde se hallan inscritas las claves de la formación, y llegar a una visión crítica e intercultural que permita el diálogo entre universidad y comunidad, para dar sentido al ejercicio académico desde el cuestionamiento de las desigualdades y la creación de una forma alterna para postular el saber con el aporte de los pueblos originarios, dado que el academicismo restringe el acceso de las minorías étnicas a otros contextos pluriculturales; de ahí la necesidad de repensar, en el siglo $\mathrm{XXI}$, estas relaciones entre culturas, territorios, prácticas tradicionales, educación e investigación.

En ese orden de ideas, el gran desafío que se propone a la academia y las instituciones de educación superior es dar respuesta a las realidades y particularidades culturales que se viven en los diferentes lugares y contextos diversos de los países latinoamericanos, desde la consolidación de proyectos sociales e investigativos que permitan entrar en contacto con otras cosmovisiones, otras creencias, otras tradiciones, atravesadas por la palabra, la naturaleza $\mathrm{y}$, por supuesto, la vida, para reconocerlas y visibilizarlas, en otros escenarios que promuevan la construcción de memoria colectiva y el fortalecimiento del tejido social comunitario.

\section{LAS GEOGRAFÍAS DE LA INFANCIA EN COMUNIDADES ORIGINARIAS DE AMÉRICA LATINA}

Desde esa mirada y como respuesta a los retos que los contextos socioculturales colombianos, brasileños y latinoamericanos, le demandan a la academia desde los saberes originarios que 
necesitan ser visibilizados para comprender las realidades diversas, especialmente, de nuestros niños y niñas, la Fundación Universitaria del Área Andina (Colombia) y la Universidad Federal Juiz de Fora (Brasil), a través de sus Facultades de Educación y los grupos de investigación Kompetenz y GRUPEGI ${ }^{5}$, respectivamente, pusieron en marcha un ejercicio investigativo entorno a "Geografías del cuidado y la crianza desde la experiencia educativa en comunidades originarias de América Latina"; una apuesta que busca trascender las concepciones biologicistas y psicológicas que han guiado los discursos de la infancia para aportar en la construcción del pensamiento latinoamericano desde nuevas perspectivas - la geografía de la infancia, la sociología de la infancia, la antropología de la infancia, el protagonismo infantil, la participación infantil, entre otros- que impacten los contextos socioculturales diversos de los países vinculados y los procesos formativos de los futuros educadores.

Esta iniciativa académica para reflexionar y proponer nuevas miradas contextualizadas con y sobre la infancia ${ }^{7}$ desde los aportes teóricos y metodológicos de la geografía de la infancia, la cual, según Lopes (2016) "busca compreender as crianças e suas infâncias, tendo como ponto de partida essas espacialidades, as configurações que dela emanam, entre as quais destacamos a paisagem, o território e o lugar, entre outros" (p.2), también pretende "trascender el fenómeno cultural étnico, a un enfoque ético, responsable y multidimensional, en el que se tejen lazos entre los sujetos, sus experiencias y los significados que estos les otorgan, sin

$5 \quad$ Grupo de Pesquisas e Estudos em Geografia da Infância (GRUPEGI), perteneciente a UFF/UFJF y coordinado por Jader Janer Moreira Lopes y Marisol Barenco de Mello.

$6 \quad$ Durante las primeras fases del proyecto (20162017) se contó con la participación de dos investigadoras vinculadas al programa de Licenciatura en Educación para la Primera Infancia de la Universidad San Buenaventura, sede Bogotá.

$7 \quad$ La investigación está estructurada en cuatro fases, desarrolladas entre el 2016-2019. Actualmente, se encuentra en la fase de resultados. la imposición de ideas ajenas a la propia cultura; es vivir con las diferencias sin anular al otro"(Cardona, Ramírez, et al, 2017, p.24), para que se puedan revelar, desde lo familiar, lo comunitario y lo institucional, las experiencias educativas de los niños y las niñas en sus primeros años, a través del cuidado y la crianza, en comunidades originarias de América Latina, y construir, a partir de esas experiencias, esas espacialidades y esas identidades, referentes que orienten proyectos para la educación inicial, en diferentes contextos.

Justamente, esa necesidad de "visibilizar» ciertos colectivos y contextos que permanecen «invisibilizados» en la geografía humana, permite trazar un trabajo investigativo que centra su interés en las prácticas sociales y espaciales, para analizar desde el cuidado y la crianza, esas infancias que habitan el territorio latinoamericano, ya que "Dada a diversidade de contextos sociais e a pluralidade da sociedade, precisamos reconhecer a existência de diferentes infâncias que acolhem diferentes crianças com histórias de vidas distintas, com sentidos e significados peculiares"(Verbena, 2016, p.2).

Los protagonistas de este proyecto de investigación son dos comunidades originarias, una colombiana y una brasileña; una indígena y una afrodescendiente; dos grupos humanos que abrieron las puertas de sus territorios y sus almas para realizar una travesía comunitaria por sus saberes ancestrales, sus prácticas tradicionales, sus lugares, sus paisajes; en suma, sus cosmovisiones y sus identidades culturales; unas maneras particulares de ver, ser y actuar en el mundo, que revelan las experiencias y vivencias de los más pequeños: los niños y las niñas de la Comunidad Embera Katío de Frontino, Antioquia -Colombia- y la Comunidad Quilombola Colônia do Paiol de Bias Fortes, Minas Gerais -Brasil-. 


\section{COMUNIDADES EMBERA KATÍO Y QUILOMBOLA COLÔNIA DO PAIOL}

Los Embera Katío son uno de los cuatro pueblos Embera que existen en Colombia. En lengua eyabida su nombre significa "Habitantes de Montaña". De acuerdo con el Censo del DANE en 2005, en el país son 38.259 indígenas Embera Katío. Este pueblo ${ }^{8}$ representa el $2.7 \%$ de la población indígena de Colombia. En su mayoría, se encuentran ubicados en los epartamentos de Antioquia, Chocó y Córdoba; Antioquia concentra el $33.5 \%$ de la población.

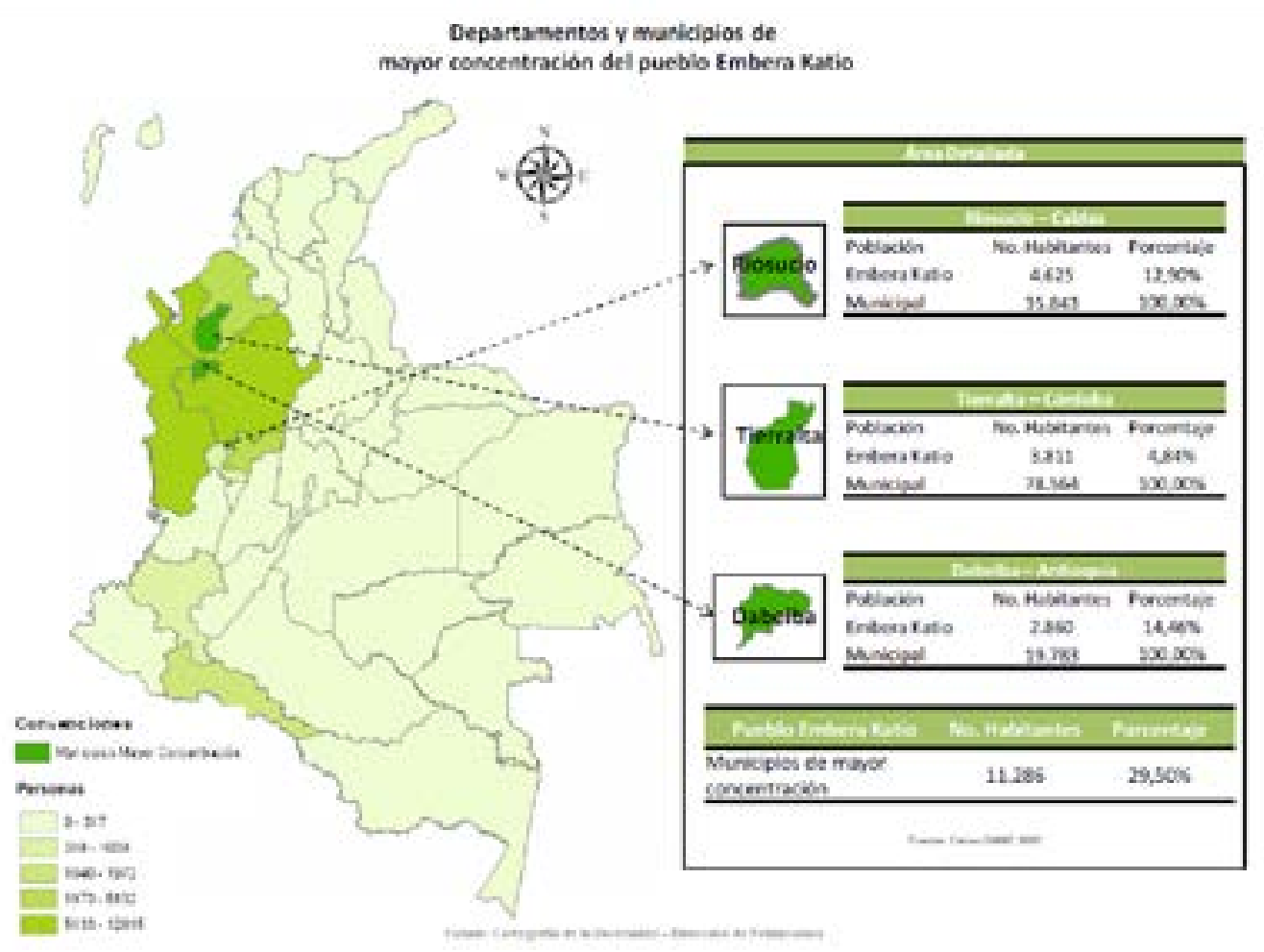

Figura 1. Ubicación geográfica y demográfica de los Embera Katío. Fuentes: Cartografía de la Diversidad- Dirección de Poblaciones. Disponible en: http://observatorioetnicocecoin.org.co/cecoin/ files/Caracterizaci\%C3\%B3n\%20del\%20pueblo\%20Embera\%20Kat\%C3\%ADo. pdf

\footnotetext{
$8 \quad$ En 2009, la Corte Constitucional Colombiana, en su Auto 004, los declaró en Riesgo y ordenó al Estado "la construcción de un Programa de Garantía de los Derechos de los Pueblos Indígenas afectados por el desplazamiento y además la elaboración y puesta en marcha de 34 planes de salvaguarda para igual número de pueblos, por considerar que se encuentran en riesgo de extinción física y cultura".
}

Justamente, en Antioquia se encuentra ubicado Frontino, un municipio a donde llega, una vez al mes y desde diferentes lugares de lazona rural, un grupo de estudiantes libres e indígenas Embera Katío que cursan el programa de Licenciatura en Pedagogía Infantil de la Fundación Universitaria del Área Andina y quienes son protagonistas de 
este ejercicio investigativo. La puesta en marcha de este proceso de formación profesional ha representado para la Institución y la Licenciatura en Pedagogía Infantil,

una manera de vivenciar el componente misional de Responsabilidad Social de la Institución, y en consecuencia, la oportunidad de generar, un diálogo de saberes, en torno al sentido de la formación de maestros desde los diferentes contextos socioculturales que demandan al ejercicio del quehacer docente, una formación de los niños y las niñas desde las particularidades de sus comunidades". (Cardona, Ramírez, et al, 2017, p.18)

La mayoría de ellos recorren grandes distancias a pie, caballo, chiva o bus, para adelantar sus estudios profesionales en Pedagogía Infantil; un proceso formativo que se ha construido desde el respeto y el reconocimiento de los saberes tradicionales y culturales, y que posteriormente, compartirán en cada una de sus comunidades y sus resguardos. Una experiencia significativa que se teje desde la comunidad, las prácticas pedagógicas, la educación de los niños y las niñas, el diálogo intercultural, el pasado y el presente, como espacios de reconocimiento y reencuentro.

En relación con la Comunidad Quilombola existen diversas conceptualizaciones respecto a su origen y sus características; algunas de esas perspectivas son excluyentes y limitan la comprensión de ese grupo humano. Para el caso de esta investigación y el contexto brasileño, los Quilombos se entienden como "uma organização social e de experiência coletiva de africanos e seus descendentes, como forma de reação e resistência ao processo de escravidão" (Verbena, 2016, p.6), ya que actualmente, la noción de Quilombos va más allá de comunidades formadas exclusivamente por antiguos esclavos y se asume como: condição, essa, conquistada por reivindicações e lutas políticas dos quilombolas, amparadas em pesquisas sobre a temática. Reconhecer e ampliar o entendimento de comunidades quilombolas como aquelas formadas por descendentes de escravos significa dar visibilidade à história, à cultura e ao papel político exercido pelos quilombos, valorizando a memória afro-brasileira e combatendo o racismo existente na sociedade. (Verbena, 2016, p.6)

Según el artículo 20 del Decreto 4887 del 20 de noviembre de 2003 de la Presidencia de la República de Brasil, las Comunidades Quilombolas se consideran "os grupos étnicoraciais, segundo critérios de auto-atribuição, com trajetória histórica própria, dotados de relações territoriais específicas, com presunção de ancestralidade negra relacionada com a resistência à opressão histórica sofrida" (Presidência da República Federativa do Brasil, 2003).

En2004selanzó"Brasil Quilombola”, un programa que tiene como objetivo consolidar las políticas estatales para las áreas donde se encuentran estas comunidades, a partir de cuatro pilares: acceso a la tierra; infraestructura y calidad de vida; inclusión productiva y desarrollo local; así como derechos y ciudadanía. De acuerdo con la Fundación Cultural Palmares ${ }^{9}$ (FCP), vinculada al Ministerio de Cultura de Brasil, desde su creación en 1988, han certificado a 2962 Comunidades Quilombolas ${ }^{10}$, las cuales se encuentran ubicadas en casi todos los 9 Según la Fundação Cultural Palmares (FCP) "No Brasil, o termo quilombo é utilizado para nomear os locais de refúgio e resistência dos escravos fugidos durante os períodos colonial e imperial. As comunidades que então se formaram também incluíram, além dos ex-escravos negros, indígenas, mestiços e brancos pobres. O mais famoso deles, o Quilombo do Palmares, foi homenageado ao dar seu nome à $F C P$, criada para promover e preservar a arte e a cultura afro-brasileira". (Disponible en: http://www.palmares. gov.br)

10 Ese reconocimiento es el primer paso para que inicien el proceso de titulación de tierras ante el INCRA- Instituto Nacional de Colonização e Reforma Agrária-. 
Estados de Brasil; sin embargo, el mayor número de ellas se ubican en: Bahía (718), Marañón (653), Minas Gerais (275), Pará (245) y Pernambuco (138). ${ }^{11}$

\section{Número de Comunidades Remanescentes de Quilombos por Estado}

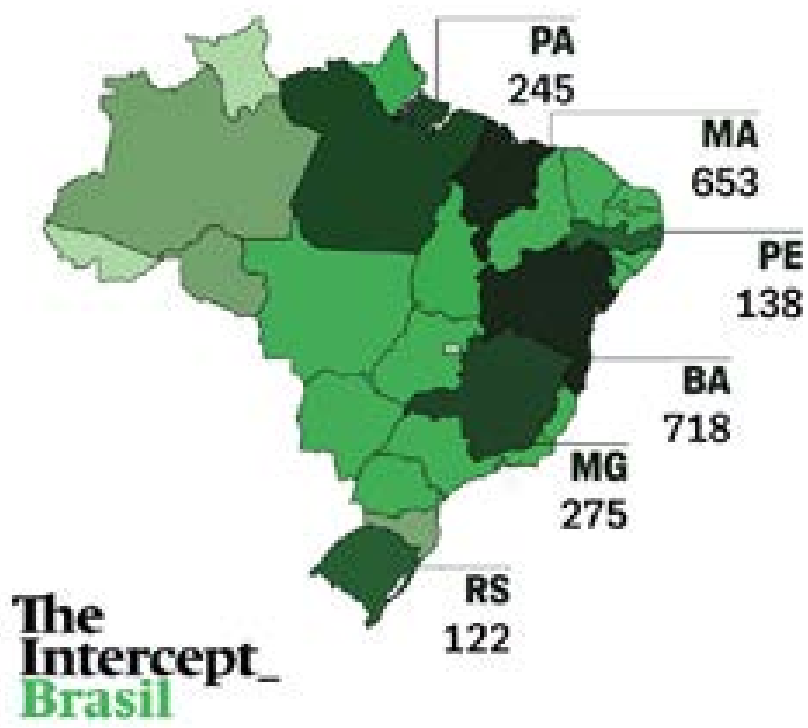

\begin{tabular}{|lrlr} 
BA 718 & PB & 38 \\
MA 653 & PR & 37 \\
MG 275 & RJ & 35 \\
PA 245 & SE & 35 \\
PE 138 & G0 & 33 \\
RS 122 & MS & 22 \\
PI & 86 & RN & 22 \\
MT & 71 & SC & 13 \\
AL & 68 & AM & 8 \\
SP & 54 & RO & 8 \\
CE & 46 & AC & 8 \\
TO & 44 & DF & 6 \\
AP & 40 & RR & 8 \\
ES & 38 & & \\
& &
\end{tabular}

Figura 2. Comunidades Remanescentes de Quilombolos por Estados Brasileños. En: Gonçalves, J. y Dezan, T. (2017). Disponible en: https://theintercept.com/2017/05/12/em-pleno-seculo-xxiquilombolas-ainda-tem-que-lutar-por-direitos-basicos/

Precisamente, en el Estado de Minas Gerais, municipio de Bias Forte, se encuentra la Colônia do Paiol, una comunidad Quilombola de 600 habitantes, certificada por la Fundación Cultural de Palmares en 2007, pero no reconocida por el gobierno de esa ciudad; una situación que según sus residentes, "influencia negativamente a juventude, a construção de sua identidade étnica e quilombola, e a educação, que não é organizada de forma diferenciada para atender às especificidades de comunidades dessa natureza, conforme estabelecem as Diretrizes Curriculares Nacionais da Educação Escolar Quilombola" (Verbena, 2016, p.10).

$11 \quad$ Fuente: Fundación Cultural Palmares. Información actualizada 28 de mayo de 2016.
La Colônia do Paiol ha sido estudiada por investigadores de la Universidad Federal de Juiz de Fora, especialmente, por el Núcleo de Investigación de Geografía, Espacio y Acción (NuGea), por sus siglas en portugués; esos estudios y su enfoque de trabajo, con comunidades afrodescendientes, son un antecedente fundamental para la realización de este proyecto de investigación que involucra una Comunidad Quilombola brasileña y una Comunidad Indígena colombiana.

\section{INFANCIA(S), CUIDADO Y CRIANZA}

Cuando de Rousseau enunció que el niño no es un hombre pequeño, cimentó las bases para lo 
que sería la reinvención moderna de la infancia y su concreción en el siglo XX, período en el cual, la infancia fue explícitamente reconocida como una etapa de la vida con características y necesidades propias, y el niño fue visto como persona con derecho a la identidad personal, la dignidad y la libertad.

La reinvención moderna de la infancia, tal como lo expresa Leonor Jaramillo (2007) obedece al cambio histórico y cultural que ha tenido su concepción; así, el niño pasó de ser considerado como dependiente (Siglos IV y V), propiedad de los adultos (Siglo XVI), humano inacabado, inocente y bondadoso (Siglos XVI-XVIII) a sujeto social de derecho (Siglos XX-XXI) diversificado en su expresión niño y niña.

Esta evolución evidenció la lucha por ampliar el estatus de ciudadanía, en tanto condición jurídica y material que permitiera responder a necesidades manifiestas en el bienestar de los niños y las niñas, como sujetos capaces de participar y enunciar su palabra, en lo que respecta a su experiencia vital, es decir, un sujeto libre, un sujeto de saberes, un sujeto político, un sujeto social de derechos.

Se trata entonces de instalar una nueva perspectiva sobre las infancias que dé cuenta del "mundo vivido por los niños a partir de sus espacialidades y las experiencias cotidianas, en contextos socioculturales diversos, en este caso, en la Comunidad Quilombola Colônia do Paiol y en la Comunidad indígena Embera Katío de Frontino, Antioquia"; una experiencia educativa que garantice los derechos y que tenga en todo momento la participación, la opinión y la evaluación de sus protagonistas; una experiencia educativa que cree otras estrategias u otras miradas para reinventarse e insertarse en un espacio heterogéneo, multicultural y polivalente, en el cual, se encuentran sumergidas las infancias, es decir, las nuevas generaciones de niños y niñas sujetos constructores de su realidad, de manera participativa.
Con esos propósitos, el proyecto de "Geografías del cuidado y la crianza desde la experiencia educativa en comunidades originarias deAmérica Latina", puso en escena, a través del enfoque de la geografía de la infancia, las categorías conceptuales: cuidado, crianza, comunidad originaria, comunalidad e interculturalidad, desde la experiencia educativa, las cuales han permitido avanzar en la construcción teórica, el desarrollo metodológico y el acercamiento a las comunidades originarias para explorar su formas de pensar y actuar alrededor de los niños y las niñas en sus tres primeros años.

Dada la naturaleza cualitativa de esta investigación y teniendo en cuenta el enfoque de la geografía de la infancia, se han asumido técnicas consecuentes que permiten hacer una ruta de encuentro sobre la experiencia educativa, en donde la memoria, el rostro, la palabra y el territorio emerjan, a partir de las categorías propuestas. De este modo, las técnicas asumidas para el trabajo de campo con las comunidades han sido: notas de campo, diarios de campo, cartogramas, cartografía social, cartografías culturales, mapas vivenciales, ${ }^{12}$ narrativas, fotografías, entre otros.

12 Lopes, J. J. M. (2012). Mapas narrativos e espaços vivenciais: cartografando os lugares de infância. En $D$. Andrade y J. Lopes. (Orgs). Infâncias e crianças: lugares em diálogos. Cuiabá, Brasil: UFMT. 


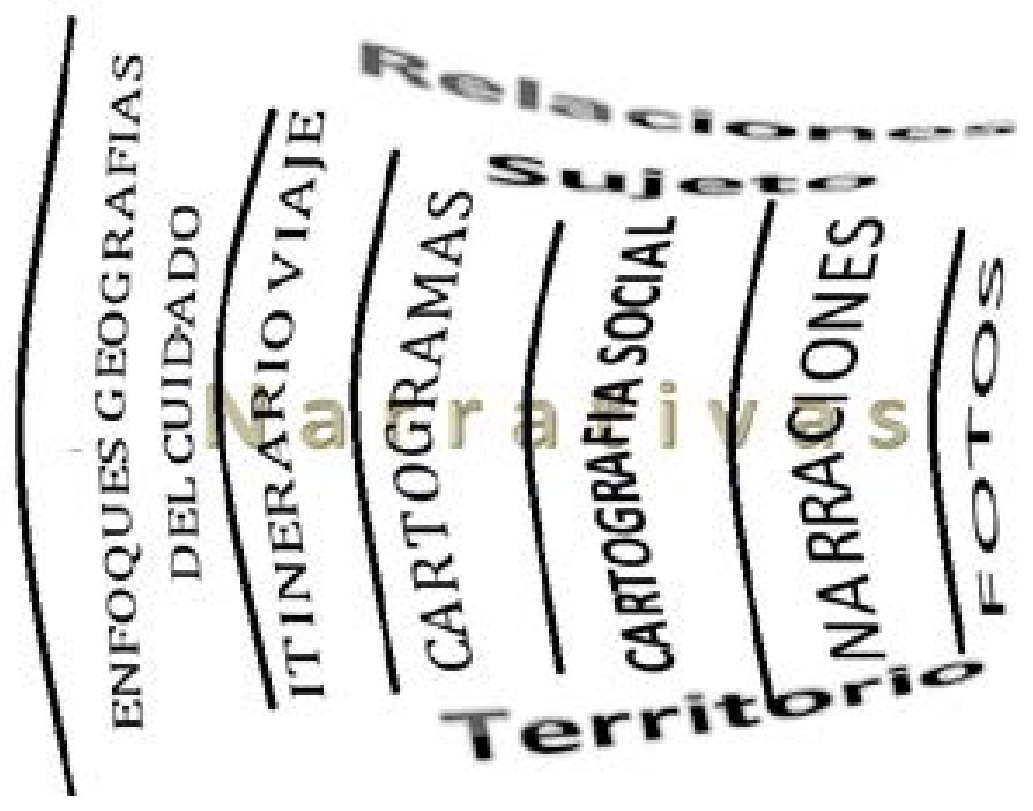

Figura 3. Mapa metodológico y técnicas usadas en el desarrollo del Proyecto de Geografías del cuidado y la crianza.

Sin embargo, el ejercicio investigativo no se ha quedado solo en las técnicas, en la construcción teórica de las categorías, en el trabajo de campo que cada grupo de investigadores hace en Colombia o Brasil con las comunidades originarias Embera Katío y Quilombola Colônia do Paiol, respectivamente; tampoco se ha limitado a las reflexiones de los investigadores vinculados a este proyecto que se reúnen frecuentemente, sino que ha trascendido las fronteras geográficas y lingüísticas para propiciar encuentros presenciales que nutran esta experiencia, en el marco de la internacionalización de Educación Superior, entendida como:

un proceso que fomenta los lazos de cooperación e integración de las Instituciones de Educación Superior (IES) con sus pares en otros lugares del mundo, con el fin de alcanzar mayor presencia $y$ visibilidad internacional en un mundo cada vez más globalizado. Este proceso le confiere una dimensión internacional e intercultural a los mecanismos de enseñanza e investigación de la educación superior, a través de la movilidad académica de estudiantes, docentes e investigadores; la formulación de programas de doble titulación; el desarrollo de proyectos conjuntos de investigación y la internacionalización del currículo; así como la conformación de redes internacionales y la suscripción de acuerdos de reconocimiento mutuo de sistemas de aseguramiento de la calidad de la educación superior, entre otros. (MEN, 2009)

\section{MISIONES ACADÉMICAS INTERNACIONALES DE INVESTIGACIÓN Y EDUCACIÓN}

Justamente, esa dimensión internacional e intercultural de la investigación, en este caso, se ha potencializado a través de tres Misiones Académicas Internacionales de Investigación y Educación tanto en Brasil como en Colombia para aportar en esa línea a las IES vinculadas en esta propuesta de investigación.

¿La primera Misión Académica Internacional de Investigación y Educación 
Brasil- Colombia, en el marco del proyecto de Geografías del cuidado y la crianza desde la experiencia educativa en comunidades originarias de América Latina, se realizó a la Universidad Federal de Juiz de Fora de Brasil, del 22 al 29 de octubre de 2017. La Misión incluyó un Seminario de Pesquisa programado por GRUPEGI, trabajo interno de los grupos de investigación: Colombia- Brasil; visitas a Creches, Escolas y Colegios; visitas a Centros de apoyo de la Prefectura de Juiz de Fora; participación en eventos de extensión con docentes del municipio; encuentros con la secretaria de educación y miembros de la prefectura de Juiz de Fora; así como la visita al Centro de Ciencias de la Universidad Federal de Juiz de Fora.

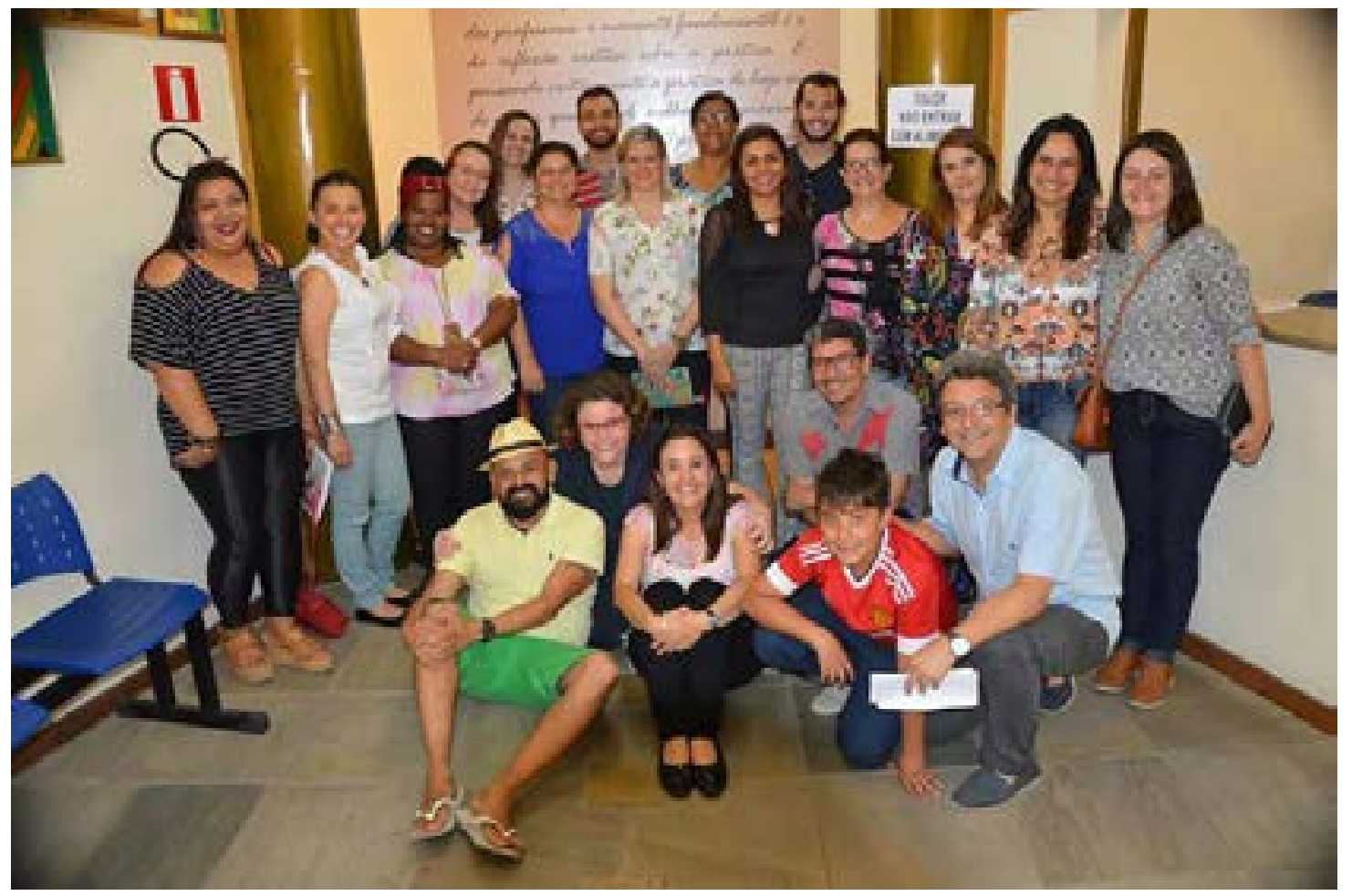

Figura 4. Misión Académica Internacional de Investigación y Educación Colombia- Brasil.

Participantes en Encuentro con el equipo de educación infantil del municipio de Juiz de Fora, Brasil.

Octubre 26 de 2017. Fuente: Prefeitura Juiz de Fora.

Esta experiencia permitió conocer el contexto $\mathrm{y}$ el proceso formativo que brinda el sistema educativo brasileño a los más pequeños; compartir las circunstancias del proyecto en cada uno de los países participantes, los avances y la consolidación del proceso de investigación desde una perspectiva internacional, a partir de una ruta de trabajo común para el desarrollo conceptual de las categorías de Cuidado, Crianza y Comunidad Originaria; también el fortalecimiento de las relaciones interinstitucionales entre la Universidad Federal
Juiz de Fora y la Fundación Universitaria del Área Andina.

La segunda Misión Académica Internacional de Investigación y Educación Colombia- Brasil se realizó a la Fundación Universitaria del Área Andina, Sede Bogotá, del 15 al 21 de octubre de 2018, en el marco del IX Congreso Internacional de Investigación Areandino, evento que acoge las iniciativas de internacionalización e investigación de nuestras Facultades, y el Poscongreso de Investigación, organizado 
por la Facultad de Educación y el grupo de investigación Kompetenz.

Este espacio académico internacional de investigación y educación incluyó conversatorios y jornadas académicas en torno a la educación, la primera infancia y la investigación en comunidades originarias; talleres de mapas vivenciales con los docentes de la Facultad de Educación de la Fundación Universitaria del
Área Andina; visitas a Instituciones Educativas Distritales: actividades culturales y una salida de campo al municipio de Frontino, Antioquia para el encuentro con los estudiantes Embera Katío y libres; un viaje de dos días que permitió el intercambio de experiencias culturales, profesionales y personales, a través de talleres, mapas vivenciales, y un diálogo intercultural genuino que impactó tanto a los estudiantes como a los investigadores brasileños $y$ colombianos.

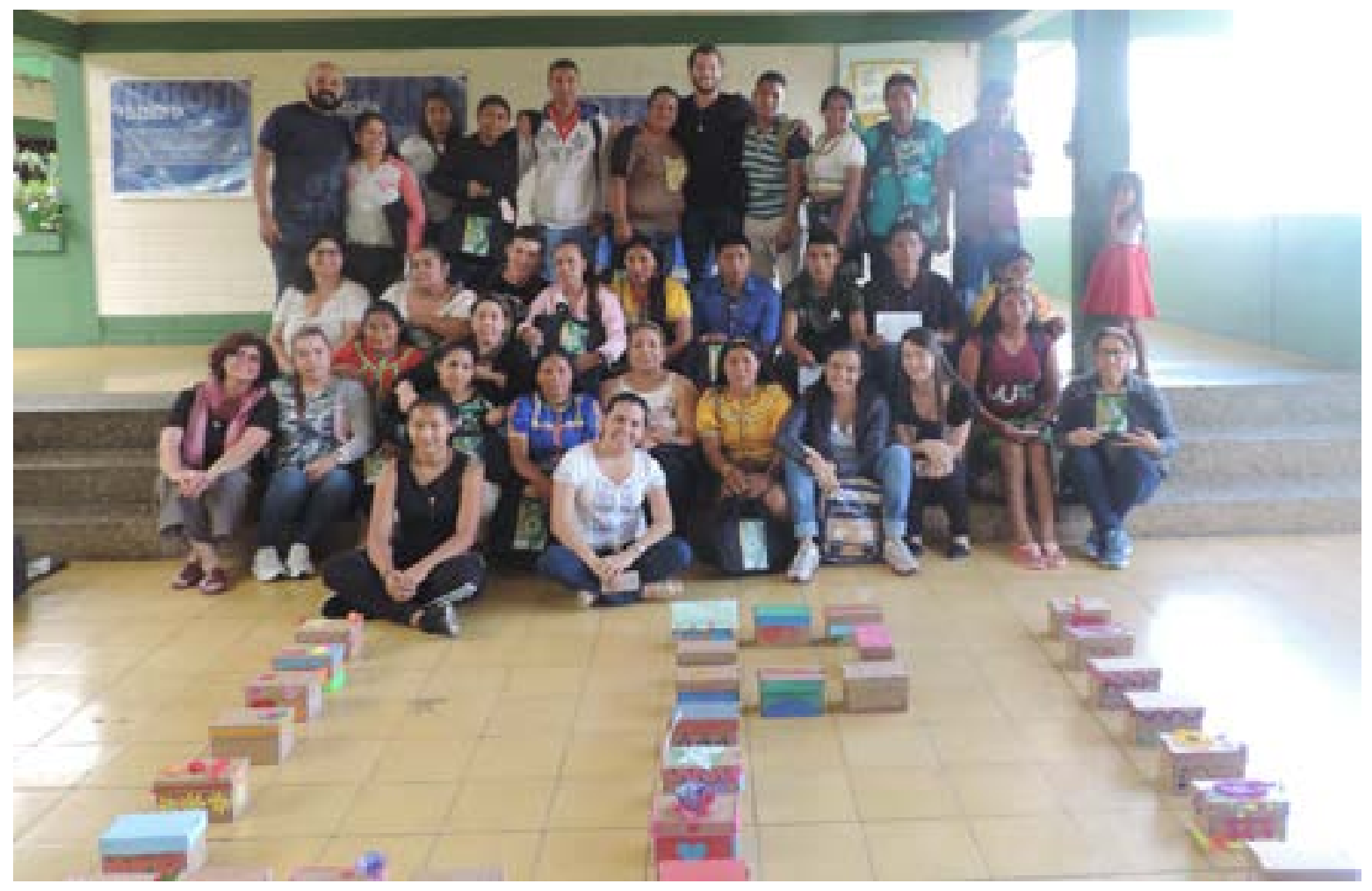

Figura 5. Misión Académica Internacional de Investigación y Educación Colombia- Brasil. Taller de cartografías y mapas vivenciales. Frontino, Antioquia. Octubre 20 de 2019.

Esa nueva oportunidad de encuentro e intercambio académico, investigativo y cultural, fortaleció y reafirmó el compromiso que la Fundación Universitaria del Área Andina y la Universidad Federal de Juiz de Fora, a través de sus grupos de investigación Kompetenz y GRUPEGI, respectivamente, y de los investigadores vinculados al proyecto de Geografías del cuidado y la crianza desde la experiencia educativa en comunidades originarias de América Latina, tienen con la(s) infancia(s)y las experiencias educativas que ellas viven en los contextos socioculturales diversos que acogen las comunidades originarias, en este caso Quilombolas y Embera Katío, desde la vida cotidiana; un vivir diverso, conversacional y sustentable que contribuya a "elaboração de conhecimentos sobre a infância, bem como para o reconhecimento e a construção de saberes necessários às experiências educacionais com 
crianças de comunidades originárias em outros alguns países da América Latina" (Verbena, 2016, p.5).

\section{A MODO DE CIERRE}

En síntesis, el contacto con otras cosmovisiones, otras creencias, otras tradiciones, atravesadas por la palabra, la naturaleza y, por supuesto, la vida, en comunidades originarias - Embera Katío y Quilombola Colônia do Paiol- de dos países latinoamericanos, representa una gran oportunidad para poner en diálogo los saberes y las experiencias en torno a las infancia(s) en las comunidades originarias desde una perspectiva internacional e intercultural, pero al mismo tiempo, es desafío para la academia, la investigación y la formación de futuros docentes de educación infantil en ambos países, ya que el contacto con los niños y las niñas, deja de ser asunto de quien cuida o acompaña, y se convierte en una condición de construcción social, que asume y reconoce al niño y la niña como parte fundamental de la sociedad; un asunto que es necesario asumir desde el mundo vivido por ellos, la experiencia educativa que se da en su cotidianidad y que no se limita a los espacios institucionales, sino a la vida en general, ya que a ellos, se les debe garantizar una infancia de calidad, un proceso de formación donde sean lo que realmente son: niños y niñas.

En este sentido, el contexto juega un papel fundamental en la constitución de la experiencia educativa, ya que permite reconocer el papel de apropiación del entorno para desarrollar relaciones en el niño $e$ ir transformado comportamientos, emociones y acciones en relación con la individualidad y la comunidad. De igual manera, es poner en horizontalidad pensamiento y acción para que los niños y las niñas desarrollen todas las dimensiones de la existencia humana, según las condiciones del contexto físico, familiar y comunitario, y las relaciones que se tejen entre sí, porque cómo señaló Wendy Dale: —"Las infancias nunca duran. Pero todo el mundo, se merece una".

\section{REFERENCIAS BIBLIOGRÁFICAS}

Cardona, L. Ramírez, M. Vergara M. Y Gutiérrez, A. (2017). Geografías del cuidado y la crianza desde la experiencia educativa en Comunidades originarias de América Latina. Bogotá, Colombia: Fundación Universitaria del Área Andina.

Corte Constitucional de la República de Colombia. (2009). Auto 004 del 26 de enero de 2009; Proteger los derechos fundamentales de las personas y los pueblos indígenas desplazados por el conflicto armado o en riesgo de desplazamiento forzado, en el marco de la superación del estado de cosas inconstitucional declarado en la sentencia T025 de 2004. Bogotá, D.C: Corte Constitucional Colombiana.

Fundación Universitaria del Área Andina. [Areandina]. (2018, junio, 13). Historias de vida Areandina. Grados Frontino 2018- Fac. De Educación [Archivo de video]. Recuperado de https://www.youtube.com/watch?v=aP9AcC8y_k

Gonçalves, J. y Dezan, T. (12 de Maio de 2017). Em pleno século $X X I$, quilombolas ainda têm que lutar por direitos básicos. The intercept Brasil. Recuperado de: https://theintercept. com/2017/05/12/em-pleno-seculo-xxiquilombolas-ainda-tem-que-lutar-por-direitosbasicos/

Jaramillo, L. (2007). Concepción de infancia. Revista del Instituto de Estudios Superiores en Educación, 8, 108-123. Páginas.

Lopes, J. J. M. (2012). Mapas narrativos e espaços vivenciais: cartografando os lugares de infância. En D. Andrade y J. Lopes. (Orgs). Infâncias e crianças: lugares em diálogos. Cuiabá, Brasil: UFMT 
Lopes, J. J. M. (2016). Geografia e educação infantil. Espaços e tempos desacostumados. Porto Alegre, Brasil: Editora Mediação.

Ministerio de Educación Nacional de Colombia. (2009). Internacionalización de la Educación Superior. Recuperado de: https://www. mineducacion.gov.co/1621/article-196472.html

Presidência da República Federativa do Brasil. (2003). Decreto 4887 de 20 de novembro de 2003: regulamenta o procedimento para identificação, reconhecimento, delimitação, demarcação e titulação das terras ocupadas por remanescentes das comunidades dos quilombos de que trata o art. 68 do Ato das Disposições Constitucionais Transitórias. Brasilia: Presidência da República Federativa do Brasil.

Verbena, E. (2016). Experiências educativas em comunidades quilombolas. En GRUPEGI. Proyecto de investigación con la Comunidad Quilombola Colônia do Paiol, en J. Moreira Lopes y E. do Carmo Garcia Verbena (coords.), Pantevis M. y E. Rodrigues (participantes). Investigación en proceso, integrada al proyecto Geografías de Cuidar y Educar: Grupo de Investigaciones y Estudios en Geografía de la Infancia (por sus siglas en portugués, GRUPEGI). [En línea]. Universidade Federal Juiz de Forauff, Minas Gerais, Brasil. Disponible en: http:// geografiadainfancia.blogspot.com.br/ 